\title{
CATEGORICAL ABSTRACT ALGEBRAIC LOGIC: SUBDIRECT REPRESENTATION OF POFUNCTORS
}

\section{George Voutsadakis}

School of Mathematics and Computer Science, Lake Superior State University, Sault Sainte Marie, Michigan, USA

Patasinska and Pigozzi developed a theory of partially ordered varieties and quasi-varieties of algebras with the goal of addressing issues pertaining to the theory of algebraizability of logics involving an abstract form of the connective of logical implication. Following their lead, the author has abstracted the theory to cover the case of algebraic systems, systems that replace algebras in the theory of categorical abstract algebraic logic. In this note, an order subdirect representation theorem for partially ordered algebraic systems is proven. This is an analog of the Order Subdirect Representation Theorem of Patasinska and Pigozzi, which, in turn, generalizes the well-known Subdirect Representation Theorem of Universal Algebra.

Key Words: Algebraic systems; Algebraizable logics; $\pi$-institutions; Order homomorphisms; Order isomorphisms; Order translations; Polarities; Polarity translations; Protoalgebraic $\pi$-institutions; Protoalgebraic logics; Quasi-varieties; Subdirect products; Subdirect representation theorem; Varieties.

2000 AMS Subject Classification: Primary 18C15, 03G99; Secondary 08C05, 08B05, 68N30.

\section{INTRODUCTION}

The present article is one of a series of articles by the author that abstracts aspects of the theory of partially ordered algebras to the level of algebraic systems. Algebraic systems replace algebras in the theory of categorical abstract algebraic logic. The motivation for this abstraction comes from investigations in the theory of abstract algebraic logic pioneered by Pałasińska and Pigozzi (see Pigozzi, Preprint) on the possibility of creating a theory of algebraization of logics involving an abstract form of the connective of implication. The traditional operator approach to the algebraization of logics (see, e.g., Blok and Pigozzi, 1986, 1989) deals with logical equivalence rather than logical implication. To Pałasińska and Pigozzi, this problem called for the passage from varieties and quasi-varieties of universal algebras, which are the main algebraizing modules in the operator approach, to ordered varieties and quasi-varieties of algebras.

Received September 1, 2005; Revised September 28, 2005. Communicated by I. Swanson.

Address correspondence to George Voutsadakis, School of Mathematics and Computer Science, Lake Superior State University, Sault Sainte Marie, MI 49783, USA; E-mail: gvoutsad@1ssu.edu 
Ordered varieties and quasi-varieties of algebras were not studied in Pigozzi (Preprint) for the first time. A bulk of previous work has paved the way for the development of the theory of Pigozzi (Preprint). Sample references include the work of Bloom (1976) on varieties of ordered algebras, Mal'cev's work $(1966,1973)$ on quasi-varieties of first-order structures, Dellunde's (1999), Dellunde and Jansana's (1996), and Elgueta's (1997, 1998) work on first-order structures defined without equality, a special case of which are the structures defined using universal Horn logic without equality, and Dunn's work $(1991,1993)$ on gaggle theory. The book on partially ordered algebraic structures by Fuchs (1963) should also be mentioned.

The author has recently abstracted the operator approach to abstract algebraic logic to cover the case of logics formalized as $\pi$-institutions (Voutsadakis, Preprint a, 2005b). It has become clear, especially in Voutsadakis (2005b), that in this categorical theory, the role played by universal algebras in the traditional context is now assumed by algebraic systems. Thus, in subsequent work Voutsadakis (Preprint c,d) inspired by Pigozzi (Preprint), some of the notions and results on ordered algebras, as pertaining to abstract algebraic logic, were also lifted to the case of ordered algebraic systems, paralleling ordered algebras in a way similar to algebraic systems paralleling ordinary universal algebras.

In the present work the Order Subdirect Representation Theorem of Pigozzi (Preprint), generalizing the well-known Subdirect Representation Theorem of Universal Alegbra (Burris and Sankappanavar, 1981, McKenzie et al., 1987), is abstracted to cover the case of ordered algebraic systems.

Recall that an $\mathscr{L}$-algebra $\mathbf{A}=\left\langle A, \mathscr{L}^{\mathbf{A}}\right\rangle$ is said to be a subdirect product of the $\mathscr{L}$-algebras $\mathbf{A}_{i}=\left\langle A_{i}, \mathscr{L}^{\mathbf{A}_{i}}\right\rangle, i \in I$, written $\mathbf{A} \leq_{\mathrm{SD}} \prod_{i \in I} \mathbf{A}_{i}$, if $\mathbf{A}$ is a subalgebra of the direct product $\prod_{i \in I} \mathbf{A}_{i}$ and, in addition, the projections $\pi_{i}: \mathbf{A} \rightarrow \mathbf{A}_{i}, i \in I$, are surjective, for all $i \in I$. On the other hand, an $\mathscr{L}$-algebra $\mathbf{A}$ is said to be subdirectly irreducible if, whenever $\mathbf{A} \cong ; \leq_{\mathrm{SD}} \prod_{i \in I} \mathbf{A}_{i}$, i.e., whenever $\mathbf{A}$ is isomorphic to a subdirect product of $\mathscr{L}$-algebras $\mathbf{A}_{i}, i \in I$, there exists an $i \in I$, such that $\mathbf{A} \cong \mathbf{A}_{i}$. The universal algebraic Subdirect Representation Theorem asserts that every $\mathscr{L}$-algebra is isomorphic to a subdirect product of subdirectly irreducible $\mathscr{L}$-algebras.

Similarly to the case of universal algebras, a partially ordered algebra $\mathscr{A}=\langle\mathbf{A}, \leq\rangle$ is an order subdirect product of a collection of partially ordered algebras $\mathscr{A}_{i}=\left\langle\mathbf{A}_{i}, \lesssim_{i}\right\rangle, i \in I$, written $\mathscr{A} \subseteq$ SD $\prod_{i \in I} \mathscr{A}_{i}$, if it is an order subalgebra of their order direct product and all projection homomorphisms are surjective. Moreover, a partially ordered algebra $\mathscr{A}$ is order subdirectly irreducible iff, whenever it is isomorphic to an order subdirect product, $\mathscr{A} \cong ; \subseteq_{\mathrm{SD}} \prod_{i \in I} \mathscr{A}_{i}$, of a collection of partially ordered algebras $\mathscr{A}_{i}, i \in I$, it must be isomorphic with one of the algebras in the collection. The Order Subdirect Representation Theorem (Theorem 2.22 of Pigozzi, Preprint) states that every partially ordered algebra is isomorphic to an order subdirect product of order subdirectly irreducible partially ordered algebras.

On the other hand, a partially ordered algebraic system or partially ordered functor (pofunctor), Voutsadakis (2006) $\langle$ SEN, $\lesssim\rangle$ is said to be an order subdirect product of the collection of pofunctors $\left\langle\mathrm{SEN}^{i}, \lesssim^{i}\right\rangle, i \in I$, if it is an order

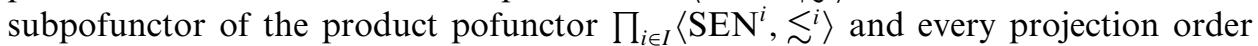
translation is surjective. The concept of an order subdirectly irreducible pofunctor differs slightly from that of an order subdirectly irreducible ordered algebra. More precisely, a pofunctor $\langle\mathrm{SEN}, \lesssim\rangle$ will be said to be order subdirectly irreducible if, 
whenever it is order isomorphic with an order subdirect product of a collection $\left\langle\mathrm{SEN}^{i}, \lesssim^{i}\right\rangle, i \in I$, written $\langle\mathrm{SEN}, \lesssim\rangle \cong ; \subseteq_{\mathrm{SD}} \prod_{i \in I}\left\langle\mathrm{SEN}^{i}, \lesssim^{i}\right\rangle$, there exists a surjective order translation $\langle H, \gamma\rangle:\langle\mathrm{SEN}, \lesssim\rangle \rightarrow^{p}\left\langle\mathrm{SEN}^{i}, \lesssim^{i}\right\rangle$, for some $i \in I$, such that $\gamma_{\Sigma}$ : $\operatorname{SEN}(\Sigma) \rightarrow \operatorname{SEN}^{i}(H(\Sigma))$ is an order isomorphism, for all $\Sigma \in|\operatorname{Sign}|$. This deviation from insisting that there exist an order isomorphism from $\langle\mathrm{SEN}, \lesssim\rangle$ to one of the $\left\langle\mathrm{SEN}^{i}, \lesssim^{i}\right\rangle, i \in I$, is due to the more complicated signature structures that one has to deal with in this abstract framework of algebraic systems. The exact reason why this modification occurs will become more apparent after Proposition 1 has been formulated, which parallels in the framework of algebraic systems Proposition 2.20 of Pigozzi (Preprint).

For general concepts and notation from category theory, the reader is referred to any of the books Barr and Wells (1999), Borceux (1994), Mac Lane (1971). For an overview of the current state of affairs in abstract algebraic logic, the review article (Font et al., 2003), the monograph (Font and Jansana, 1996), and the book (Czelakowski, 2001) are all excellent references. To follow recent developments on the categorical side of the subject, the reader may refer to the series of articles, Voutsadakis (Preprint a,b,c,d, 2005) (see also additional references therein).

\section{SUBDIRECT REPRESENTATION THEOREM}

Let $\mathrm{SEN}^{i}: \mathbf{S i g n}^{i} \rightarrow$ Set, $i \in I$, be a collection of functors, $N^{i}, i \in I$, compatible categories of natural transformations on $\mathrm{SEN}^{i}, i \in I$, respectively, and $\rho^{i}, i \in I$, compatible polarities for $N^{i}, i \in I$, respectively. A $\prod_{i \in I} \rho^{i}$-pofunctor $\left\langle\mathrm{SEN}^{\prime}, \lesssim^{\prime}\right\rangle$ is said to be an order subdirect product of the system of $\rho^{i}$-pofunctors $\left\langle\mathrm{SEN}^{i}, \lesssim^{i}\right\rangle, i \in I$, in symbols $\left\langle\mathrm{SEN}^{\prime}, \lesssim^{\prime}\right\rangle \subseteq \mathrm{SD} \prod_{i \in I}\left\langle\mathrm{SEN}^{i}, \lesssim^{i}\right\rangle$, if:

(i) $\left\langle\mathrm{SEN}^{\prime}, \lesssim^{\prime}\right\rangle$ is a $\prod_{i \in I} \rho^{i}$-subpofunctor of $\prod_{i \in I}\left\langle\mathrm{SEN}^{i}, \lesssim^{i}\right\rangle$ and

(ii) The composite $\left\langle P^{i}, \pi^{i}\right\rangle \circ\langle J, j\rangle:\left\langle\mathrm{SEN}^{\prime}, \lesssim^{\prime}\right\rangle \rightarrow\left\langle\mathrm{SEN}^{i}, \lesssim^{i}\right\rangle$, where $\langle J, j\rangle:\left\langle\mathrm{SEN}^{\prime}\right.$, $\left.\lesssim^{\prime}\right\rangle \rightarrow^{p} \prod_{i \in I}\left\langle\mathrm{SEN}^{i}, \lesssim^{i}\right\rangle$ is the inclusion and $\left\langle P^{i}, \pi^{i}\right\rangle: \prod_{i \in I}\left\langle\mathrm{SEN}^{i}, \lesssim^{i}\right\rangle \rightarrow^{p}$ $\left\langle\mathrm{SEN}^{i}, \lesssim^{i}\right\rangle$ the projection,

$$
\left\langle\mathrm{SEN}^{\prime}, \lesssim^{\prime}\right\rangle \stackrel{\langle J, j\rangle}{\longrightarrow} \prod_{i \in I}\left\langle\mathrm{SEN}^{i}, \lesssim^{i}\right\rangle \stackrel{\left\langle P^{i}, \pi^{i}\right\rangle}{\longrightarrow}\left\langle\mathrm{SEN}^{i}, \lesssim^{i}\right\rangle
$$

is a surjective order translation, for all $i \in I$.

Given a class $\mathrm{K}$ or pofunctors, with compatible categories of natural transformations and polarities, the class of all pofunctors order isomorphic to order subdirect products of some collection of members of $\mathrm{K}$ is denoted by $\mathbf{P}_{\mathrm{SD}}(\mathrm{K})$.

A collection of functors $H^{i}: \mathbf{S i g n}^{\prime} \rightarrow \mathbf{S i g n}^{i}, i \in I$, is said to be collectively mono, if, for all $\Sigma, \Sigma^{\prime} \in\left|\mathbf{S i g n}^{\prime}\right|, H^{i}(\Sigma)=H^{i}\left(\Sigma^{\prime}\right)$, for all $i \in I$, imply that $\Sigma=\Sigma^{\prime}$,

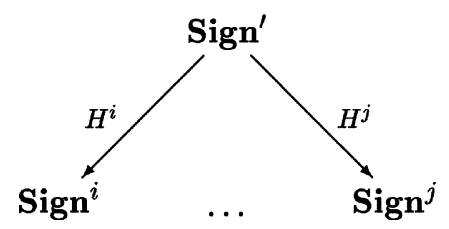


and, similarly, for all $\Sigma, \Sigma^{\prime} \in\left|\operatorname{Sign}^{\prime}\right|, f, f^{\prime} \in \operatorname{Sign}^{\prime}\left(\Sigma, \Sigma^{\prime}\right), H^{i}(f)=H^{i}\left(f^{\prime}\right)$, for all $i \in$ $I$, imply $f=f^{\prime}$. This notion will be used in the following characterization of a pofunctor being isomorphic to an order subdirect product of a given collection of pofunctors.

Proposition 1. Suppose that $\mathrm{SEN}^{i}: \mathbf{S i g n}^{i} \rightarrow \mathbf{S e t}, i \in I$, is a collection of functors, with compatible categories of natural transformations $N^{i}$ on $\mathrm{SEN}^{i}, i \in I$, and compatible polarities $\rho^{i}$ for $N^{i}, i \in I$. A $\rho$-pofunctor $\left\langle\mathrm{SEN}^{\prime}, \lesssim^{\prime}\right\rangle$ is order isomorphic to a subdirect product of the $\rho^{i}$-pofunctors $\left\langle\mathrm{SEN}^{i}, \lesssim^{i}\right\rangle, i \in I$, if and only if there exist:

(i) A collection $\lesssim^{\prime i} \in \operatorname{QoSys}_{\rho}\left(\left\langle\mathrm{SEN}^{\prime}, \lesssim^{\prime}\right\rangle\right), i \in I$, such that $\bigcap_{i \in I} \lesssim^{\prime i}=\lesssim^{\prime}$ and

(ii) A family of surjective order translations $\left\langle H^{i}, \gamma^{i}\right\rangle:\left\langle\mathrm{SEN}^{\prime}, \lesssim^{\prime}\right\rangle / \lesssim^{i} \rightarrow^{p}\left\langle\mathrm{SEN}^{i}, \lesssim^{i}\right\rangle$, for all $i \in I$, such that

$$
\begin{aligned}
& -\left\{H^{i}: i \in I\right\} \quad \text { are collectively mono and } \\
& -\gamma_{\Sigma}^{i}: \operatorname{SEN}^{\sim^{\prime i}}(\Sigma) \rightarrow \operatorname{SEN}^{i}\left(H^{i}(\Sigma)\right) \quad \text { is an order isomorphism, for all } \\
& \quad i \in I \text { and all } \Sigma \in\left|\operatorname{Sign}^{\prime}\right| .
\end{aligned}
$$

Proof. First, suppose that $\left\langle\mathrm{SEN}^{\prime}, \lesssim^{\prime}\right\rangle$ is isomorphic to a subdirect product $\langle\mathrm{SEN}, \lesssim\rangle$ of the $\left\langle\mathrm{SEN}^{i}, \lesssim^{i}\right\rangle, i \in I$, via the order isomorphism $\langle F, \alpha\rangle$ : $\left\langle\mathrm{SEN}^{\prime}, \lesssim^{\prime}\right\rangle \cong p$ SEN, $\cong^{\prime}$. Consider the composite $\left\langle G^{i}, \beta^{i}\right\rangle:=\left\langle P^{i}, \pi^{i}\right\rangle \circ\langle J, j\rangle \circ$ $\langle F, \alpha\rangle:\left\langle\mathrm{SEN}^{\prime}, \lesssim^{\prime}\right\rangle \rightarrow^{p}\left\langle\mathrm{SEN}^{i}, \lesssim^{i}\right\rangle, i \in I$,

$$
\left\langle\mathrm{SEN}^{\prime}, \lesssim^{\prime}\right\rangle \stackrel{\langle F, \alpha\rangle}{\longrightarrow}\langle\mathrm{SEN}, \lesssim\rangle \stackrel{\langle J, j\rangle}{\longrightarrow} \prod_{i \in I}\left\langle\mathrm{SEN}^{i}, \lesssim^{i}\right\rangle \stackrel{\left\langle P^{i}, \pi^{i}\right\rangle}{\longrightarrow}\left\langle\mathrm{SEN}^{i}, \lesssim^{i}\right\rangle
$$

which is a surjective order translation, since it is the composite of a surjective order translation $\left\langle P^{i}, \pi^{i}\right\rangle \circ\langle J, j\rangle$ with the isomorphism $\langle F, \alpha\rangle$. The composite $K=J \circ F$ of the monomorphism $J$ with the isomorphism $F$ is also a monomorphism.

Let $\lesssim^{\prime i}=\operatorname{Ord} \operatorname{Ker}\left(\left\langle G^{i}, \beta^{i}\right\rangle\right)$ (see Definition 11 of Voutsadakis, 2006). We have, for all $\Sigma \in\left|\mathbf{S i g n}^{\prime}\right|, \phi, \psi \in \operatorname{SEN}^{\prime}(\Sigma)$,

$$
\begin{aligned}
\phi \bigcap_{i \in I} \lesssim_{\Sigma}^{\prime i} \psi & \text { iff }(\forall i \in I)\left(\pi_{F(\Sigma)}^{i}\left(\alpha_{\Sigma}(\phi)\right) \lesssim_{P^{i}(F(\Sigma))}^{i} \pi_{F(\Sigma)}^{i}\left(\alpha_{\Sigma}(\psi)\right)\right) \\
& \text { iff } \alpha_{\Sigma}(\phi) \prod_{i \in I} \lesssim_{F(\Sigma)}^{i} \alpha_{\Sigma}(\psi) \\
& \text { iff } \alpha_{\Sigma}(\phi) \lesssim_{F(\Sigma)} \alpha_{\Sigma}(\psi) \\
& \text { iff } \phi \lesssim_{\Sigma}^{\prime} \psi .
\end{aligned}
$$

Therefore $\bigcap_{i \in I} \lesssim^{\prime i}=\lesssim^{\prime}$

For the last part, the Order Homomorphism Theorem (Theorem 15 of Voutsadakis, 2006) will be used. We have that, for all $i \in I,\left\langle G^{i}, \beta^{i}\right\rangle:\left\langle\mathrm{SEN}^{\prime}, \lesssim^{\prime}\right\rangle \rightarrow^{p}$ $\left\langle\mathrm{SEN}^{i}, \lesssim^{i}\right\rangle$ is an order translation, such that $\lesssim^{\prime} \leq \lesssim^{\prime i}$. Therefore, there exists a unique order translation $\left\langle H^{i}, \gamma^{i}\right\rangle:\left\langle\mathrm{SEN}^{\prime}, \lesssim^{\prime}\right\rangle / \lesssim^{\prime i} \rightarrow^{p}\left\langle\mathrm{SEN}^{i}, \lesssim^{i}\right\rangle$, such that the following triangle commutes, for all $i \in I$ : Thus, we have, for all $i \in I, H^{i}=G^{i}=$ $P^{i} \circ(J \circ F)=P^{i} \circ K$, whence, for all $\Sigma, \Sigma^{\prime} \in\left|\mathbf{S i g n}^{\prime}\right|$, if $H^{i}(\Sigma)=H^{i}\left(\Sigma^{\prime}\right)$, for all $i \in I$, 


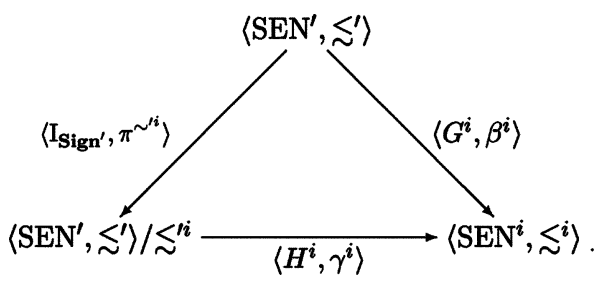

we have that $P^{i}(K(\Sigma))=P^{i}\left(K\left(\Sigma^{\prime}\right)\right)$, for all $i \in I$, and, therefore, $K(\Sigma)=K\left(\Sigma^{\prime}\right)$, and, thus, since $K$ is mono, $\Sigma=\Sigma^{\prime}$. A similar reasoning shows that, for all $\Sigma, \Sigma^{\prime} \in\left|\mathbf{S i g n}^{\prime}\right|$, $f, f^{\prime} \in \operatorname{Sign}^{\prime}\left(\Sigma, \Sigma^{\prime}\right)$, if $H^{i}(f)=H^{i}\left(f^{\prime}\right)$, for all $i \in I$, then $f=f^{\prime}$. Hence $\left\{H^{i}: i \in I\right\}$ are collectively mono. Also, for all $i \in I$ and all $\Sigma \in\left|\mathbf{S i g n}^{\prime}\right|, \phi, \psi \in \operatorname{SEN}^{\prime}(\Sigma)$,

$$
\begin{aligned}
\gamma_{\Sigma}^{i}\left(\phi / \sim_{\Sigma}^{\prime i}\right)=\gamma_{\Sigma}^{i}\left(\psi / \sim_{\Sigma}^{\prime i}\right) \quad & \text { iff } \gamma_{\Sigma}^{i}\left(\pi_{\Sigma}^{\sim^{\prime i}}(\phi)\right)=\gamma_{\Sigma}^{i}\left(\pi_{\Sigma}^{\sim^{\prime i}}(\psi)\right) \\
& \text { iff } \beta_{\Sigma}^{i}(\phi)=\beta_{\Sigma}^{i}(\psi) \\
& \text { iff } \phi \sim_{\Sigma}^{\prime i} \psi \quad\left(\text { since } \lesssim^{\prime i}=\operatorname{Ord} \operatorname{Ker}\left(\left\langle G^{i}, \beta^{i}\right\rangle\right)\right) \\
& \text { iff } \phi / \sim_{\Sigma}^{\prime i}=\psi / \sim_{\Sigma}^{\prime i} .
\end{aligned}
$$

Therefore, $\gamma_{\Sigma}^{i}$ is injective. It is also surjective since $\left\langle G^{i}, \beta^{i}\right\rangle$ is surjective, by hypothesis. Therefore $\gamma_{\Sigma}^{i}$ is a bijection, for all $i \in I$ and all $\Sigma \in\left|\mathbf{S i g n}^{\prime}\right|$.

Finally, to show that $\gamma_{\Sigma}^{i}$ is an order isomorphism, let $\Sigma \in\left|\mathbf{S i g n}^{\prime}\right|, \phi, \psi \in$ $\operatorname{SEN}^{\prime}(\Sigma)$. Then

$$
\begin{aligned}
\gamma_{\Sigma}^{i}\left(\phi / \sim_{\Sigma}^{\prime i}\right) \lesssim_{H^{i}(\Sigma)}^{i} \gamma_{\Sigma}^{i}\left(\psi / \sim_{\Sigma}^{\prime i}\right) \quad & \text { implies } \gamma_{\Sigma}^{i}\left(\pi_{\Sigma}^{\sim^{\prime i}}(\phi)\right) \lesssim_{H^{i}(\Sigma)}^{i} \gamma_{\Sigma}^{i}\left(\pi_{\Sigma}^{\sim^{\prime i}}(\psi)\right) \\
& \text { implies } \beta_{\Sigma}^{i}(\phi) \lesssim_{G^{i}(\Sigma)}^{i} \beta_{\Sigma}^{i}(\psi) \\
& \text { implies } \phi \lesssim_{\Sigma}^{\prime i} \psi \\
& \text { implies } \phi / \sim_{\Sigma}^{\prime i} \lesssim_{\Sigma}^{\prime i} / \sim_{\Sigma}^{\prime i} \psi / \sim_{\Sigma}^{\prime i} .
\end{aligned}
$$

Therefore $\gamma_{\Sigma}^{i}$ is an order isomorphism, for all $i \in I$ and all $\Sigma \in\left|\mathbf{S i g n}^{\prime}\right|$.

Suppose, conversely, that there exist:

(i) A collection $\lesssim^{\prime i} \in \operatorname{QoSys}_{\rho}\left(\left\langle\operatorname{SEN}^{\prime}, \lesssim^{\prime}\right\rangle\right), i \in I$, such that $\bigcap_{i \in I} \lesssim^{\prime i}=\lesssim^{\prime}$ and

(ii) A family of surjective order translations $\left\langle H^{i}, \gamma^{i}\right\rangle:\left\langle\mathrm{SEN}^{\prime}, \lesssim^{\prime}\right\rangle / \lesssim^{\prime i} \rightarrow^{p}$ $\left\langle\mathrm{SEN}^{i}, \lesssim^{i}\right\rangle$, for all $i \in I$, such that $\left\{H^{i}: i \in I\right\}$ are collectively mono and $\gamma_{\Sigma}^{i}: \operatorname{SEN}^{\prime \prime i}(\Sigma) \rightarrow \operatorname{SEN}^{i}\left(H^{i}(\Sigma)\right)$ is an order isomorphism, for all $i \in I$ and all $\Sigma \in\left|\mathbf{S i g n}^{\prime}\right|$.

Consider, for all $i \in I$, the order projections $\left\langle\mathrm{I}_{\mathrm{Sign}^{\prime}}, \pi^{\sim^{\prime i}}\right\rangle:\left\langle\mathrm{SEN}^{\prime}, \lesssim^{\prime}\right\rangle \rightarrow^{p}$ $\left\langle\mathrm{SEN}^{\prime}, \lesssim^{\prime}\right\rangle / \lesssim^{\prime i}$. Compose with $\left\langle H^{i}, \gamma^{i}\right\rangle$ to obtain the order translations $\left\langle H^{i}, \gamma^{\prime i}\right\rangle:\left\langle\mathrm{SEN}^{\prime}, \lesssim^{\prime}\right\rangle \rightarrow^{p}\left\langle\mathrm{SEN}^{i}, \lesssim^{i}\right\rangle, \quad i \in I$. Finally, set $\langle K, \kappa\rangle:=\prod_{i \in I}\left\langle H^{i}, \gamma^{\prime i}\right\rangle:$ $\left\langle\mathrm{SEN}^{\prime}, \lesssim^{\prime}\right\rangle \rightarrow^{p} \prod_{i \in I}\left\langle\mathrm{SEN}^{i}, \lesssim^{i}\right\rangle$. It will be shown that $\langle K, \kappa\rangle$ is an injection, whence $\left\langle\mathrm{SEN}^{\prime}, \lesssim^{\prime}\right\rangle$ is isomorphic to a subfunctor of the direct product of $\left\langle\mathrm{SEN}^{i}, \lesssim^{i}\right\rangle$ and that the composition $\left\langle P^{i}, \pi^{i}\right\rangle \circ\langle K, \kappa\rangle$ is surjective, for all $i \in I$. This will show that $\left\langle\mathrm{SEN}^{\prime}, \lesssim^{\prime}\right\rangle$ is isomorphic to a subdirect product of $\left\langle\mathrm{SEN}^{i}, \lesssim^{i}\right\rangle, i \in I$. 


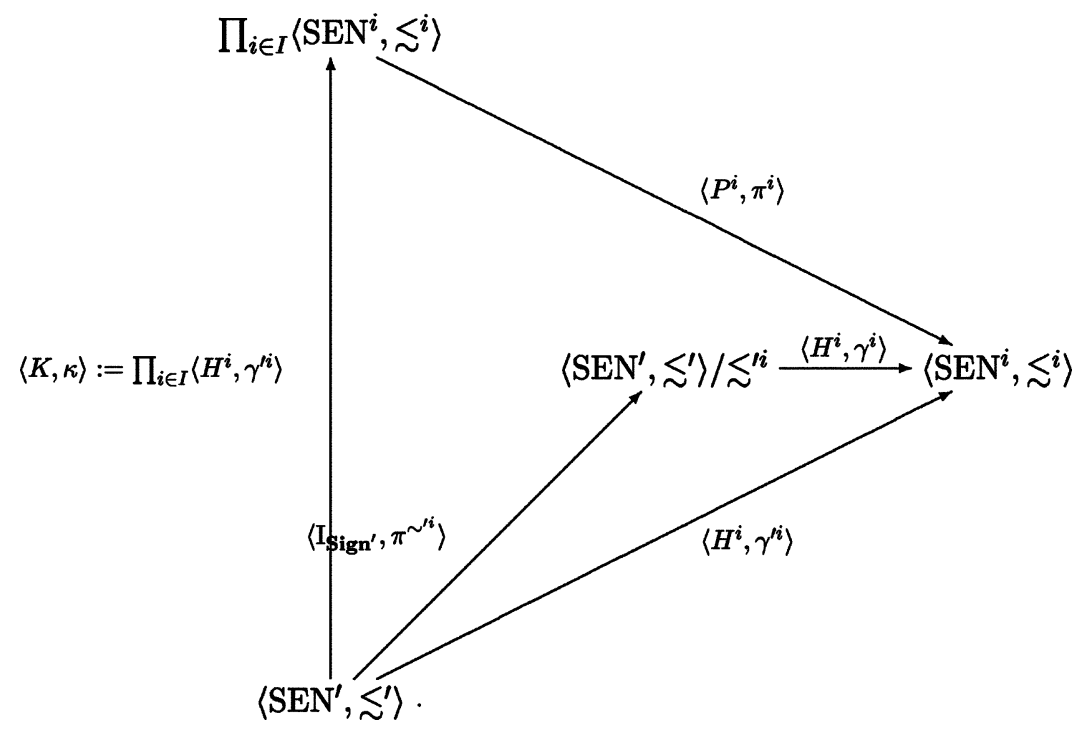

To this end, we show, first, that $\langle K, \kappa\rangle:\left\langle\mathrm{SEN}^{\prime}, \lesssim^{\prime}\right\rangle \rightarrow^{p} \prod_{i \in I}\left\langle\mathrm{SEN}^{i}, \lesssim^{i}\right\rangle$ is a monomorphism, second, that $\kappa_{\Sigma}\left(\lesssim_{\Sigma}^{\prime}\right)=\prod_{i \in I} \lesssim_{K(\Sigma)}^{i} \cap \kappa_{\Sigma}\left(\operatorname{SEN}^{\prime}(\Sigma)\right)^{2}$, for all $\Sigma \in\left|\mathbf{S i g n}^{\prime}\right|$, and finally, that $\left\langle P^{i}, \pi^{i}\right\rangle \circ\langle K, \kappa\rangle:\left\langle\mathrm{SEN}^{\prime}, \lesssim^{\prime}\right\rangle \rightarrow^{p}\left\langle\mathrm{SEN}^{i}, \lesssim^{i}\right\rangle$ is surjective, for all $i \in I$.

First, for the injectivity of $K$ on objects, we have, for all $\Sigma, \Sigma^{\prime} \in\left|\mathbf{S i g n}^{\prime}\right|$,

$$
\begin{aligned}
K(\Sigma)=K\left(\Sigma^{\prime}\right) \quad & \text { implies }(\forall i \in I)\left(P^{i}(K(\Sigma))=P^{i}\left(K\left(\Sigma^{\prime}\right)\right)\right) \\
& \text { iff }(\forall i \in I)\left(H^{i}(\Sigma)=H^{i}\left(\Sigma^{\prime}\right)\right) \\
& \text { implies } \Sigma=\Sigma^{\prime}
\end{aligned}
$$

The injectivity of $K$ on morphisms is proven similarly. For the injectivity of $\kappa_{\Sigma}: \operatorname{SEN}^{\prime}(\Sigma) \rightarrow \prod_{i \in I} \operatorname{SEN}^{i}(K(\Sigma)), \Sigma \in\left|\operatorname{Sign}^{\prime}\right|$, we have, for all $\phi, \psi \in \operatorname{SEN}^{\prime}(\Sigma)$,

$$
\begin{aligned}
\kappa_{\Sigma}(\phi)=\kappa_{\Sigma}(\psi) \quad & \text { iff } \prod_{i \in I} \gamma_{\Sigma}^{\prime i}(\phi)=\prod_{i \in I} \gamma_{\Sigma}^{\prime i}(\psi) \\
& \text { iff }(\forall i \in I)\left(\gamma_{\Sigma}^{\prime i}(\phi)=\gamma_{\Sigma}^{\prime i}(\psi)\right) \\
& \text { iff }(\forall i \in I)\left(\gamma_{\Sigma}^{i}\left(\phi / \sim_{\Sigma}^{\prime i}\right)=\gamma_{\Sigma}^{i}\left(\psi / \sim_{\Sigma}^{\prime i}\right)\right) \\
& \text { iff }(\forall i \in I)\left(\phi / \sim_{\Sigma}^{\prime i}=\psi / \sim_{\Sigma}^{\prime i}\right) \\
& \text { iff } \phi \sim_{\Sigma}^{\prime} \psi \\
& \text { iff } \phi=\psi .
\end{aligned}
$$

To show that $\kappa_{\Sigma}\left(\lesssim_{\Sigma}^{\prime}\right)=\prod_{i \in I} \lesssim_{K(\Sigma)}^{i} \cap \kappa_{\Sigma}\left(\operatorname{SEN}^{\prime}(\Sigma)\right)^{2}$, for all $\Sigma \in\left|\mathbf{S i g n}^{\prime}\right|$, suppose that $\Sigma \in\left|\operatorname{Sign}^{\prime}\right|$ and $\phi, \psi \in \operatorname{SEN}^{\prime}(\Sigma)$. We have

$$
\begin{aligned}
\phi \lesssim_{\Sigma}^{\prime} \psi \quad & \text { iff }(\forall i \in I)\left(\phi / \sim_{\Sigma}^{\prime i} \lesssim_{\Sigma}^{\prime i} / \sim_{\Sigma}^{\prime i} \psi / \sim_{\Sigma}^{\prime i}\right) \\
& \text { iff }(\forall i \in I)\left(\gamma_{\Sigma}^{i}\left(\phi / \sim_{\Sigma}^{\prime i}\right) \lesssim_{\Sigma}^{i} \gamma_{\Sigma}^{i}\left(\psi / \sim_{\Sigma}^{\prime i}\right)\right) \\
& \text { iff }(\forall i \in I)\left(\gamma_{\Sigma}^{\prime i}(\phi) \lesssim_{\Sigma}^{i} \gamma_{\Sigma}^{\prime i}(\psi)\right)
\end{aligned}
$$




$$
\begin{aligned}
& \text { iff }(\forall i \in I)\left(\pi_{\prod_{i \in I} H^{i}(\Sigma)}\left(\prod_{i \in I} \gamma_{\Sigma}^{\prime i}(\phi)\right) \lesssim_{\Sigma}^{i} \pi_{\prod_{i \in I}^{i} H^{i}(\Sigma)}\left(\prod_{i \in I} \gamma_{\Sigma}^{\prime i}(\psi)\right)\right) \\
& \text { iff } \prod_{i \in I} \gamma_{\Sigma}^{\prime i}(\phi) \prod_{i \in I} \lesssim_{\prod_{i \in I}^{i} H^{i}(\Sigma)} \prod_{i \in I} \gamma_{\Sigma}^{\prime i}(\psi) \\
& \text { iff } \kappa_{\Sigma}(\phi) \prod_{i \in I} \lesssim_{i(\Sigma)}^{i} \kappa_{\Sigma}(\psi) .
\end{aligned}
$$

Finally, to show that $\left\langle P^{i}, \pi^{i}\right\rangle \circ\langle K, \kappa\rangle:\left\langle\mathrm{SEN}^{\prime}, \lesssim^{\prime}\right\rangle \rightarrow^{p}\left\langle\mathrm{SEN}^{i}, \lesssim^{i}\right\rangle$ is surjective, for all $i \in I$, it suffices to observe that, since $\left\langle H^{i}, \gamma^{i}\right\rangle, i \in I$, are surjective, $\left\langle H^{i}, \gamma^{i}\right\rangle, i \in I$, are also surjective, and, therefore, $\left\langle P^{i}, \pi^{i}\right\rangle \circ\langle K, \kappa\rangle, i \in I$, are also surjective.

A $\rho$-pofunctor $\langle\mathrm{SEN}, \lesssim\rangle$ is order subdirectly irreducible if, whenever it is order isomorphic with an order subdirect product of a collection $\left\langle\mathrm{SEN}^{i}, \lesssim^{i}\right\rangle, i \in I$, denoted $\langle\mathrm{SEN}, \lesssim\rangle \cong ; \subseteq \mathrm{SD} \prod_{i \in I}\left\langle\mathrm{SEN}^{i}, \lesssim_{i}\right\rangle$, we have that, there exists a surjective order translation $\langle H, \gamma\rangle:\langle\mathrm{SEN}, \lesssim\rangle \rightarrow^{p}\left\langle\mathrm{SEN}^{i}, \lesssim^{i}\right\rangle$, for some $i \in I$, such that $\gamma_{\Sigma}$ : $\operatorname{SEN}(\Sigma) \rightarrow \operatorname{SEN}^{i}(H(\Sigma))$ is an order isomorphism, for all $\Sigma \in|\operatorname{Sign}|$.

The last result of the section is an analog of the Order Subdirect Representation Theorem 2.22 of Pigozzi (Preprint) for partially ordered algebraic systems. Its proof is very similar to the proof of Theorem 2.22 of Pigozzi (Preprint) but relies on Proposition 1 rather than on its analog, Proposition 2.20 of Pigozzi (Preprint).

Theorem 2 (Order Subdirect Representation Theorem). Every pofunctor is order isomorphic to an order subdirect product of order subdirectly irreducible pofunctors with compatible categories of natural transformations and compatible polarities for them.

Proof. Suppose that $\langle\mathrm{SEN}, \lesssim\rangle$ is a $\rho$-pofunctor. For all $\Sigma_{0} \in \mid$ Sign $\mid$, $\phi, \psi \in \operatorname{SEN}\left(\Sigma_{0}\right)$, such that $\phi \mathbb{Z}_{\Sigma_{0}} \psi$, there exists, by Zorn's Lemma, a maximal

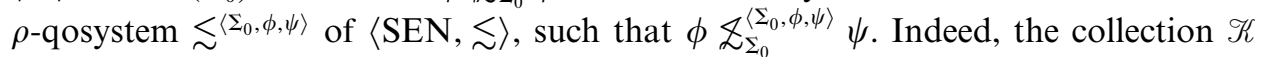
of all $\rho$-qosystems $\lesssim$ of $\left\langle\right.$ SEN, $\lesssim$, such that $\phi \grave{L}_{\Sigma_{0}}^{\prime} \psi$, is nonempty, since $\lesssim \in \mathscr{K}$. Moreover, if $\left\{\lesssim^{i}: i \in I\right\}$ is a chain in $\mathscr{H}$, it is clear that $\bigcup_{i \in I} \lesssim^{i}$ is a $\rho$-qosystem of $\langle$ SEN, $\lesssim\rangle$ and also that $\langle\phi, \psi\rangle \notin \bigcup_{i \in I} \lesssim_{\Sigma_{0}}$. Thus $\bigcup_{i \in I} \lesssim^{i}$ is an upper bound of $\left\{\lesssim^{i}: i \in I\right\}$ in $\mathscr{K}$, whence, by Zorn's Lemma, $\mathscr{K}$ has a maximal element $\lesssim\left\langle\Sigma_{0}, \phi, \psi\right\rangle$.

Now $\lesssim\left\langle\Sigma_{0}, \phi, \psi\right\rangle$ is completely meet-irreducible in the lattice $\operatorname{QoSys}_{\rho}(\langle\operatorname{SEN}, \lesssim\rangle)$. In fact, if $\lesssim^{i} \in \operatorname{QoSys}_{\rho}(\langle\mathrm{SEN}, \lesssim\rangle)$, such that $\lesssim^{\left\langle\Sigma_{0}, \phi, \psi\right\rangle}<\lesssim^{i}$, for all $i \in I$, then, by the maximality of $\lesssim\left\langle\Sigma_{0}, \phi, \psi\right\rangle$, we get that $\phi \lesssim_{\Sigma_{0}}^{i}$, for all $i \in I$, whence $\phi \bigcap_{i \in I} \lesssim \Sigma_{\Sigma_{0}} \psi$ and, therefore, $\lesssim\left\langle\Sigma_{0}, \phi, \psi\right\rangle \neq \bigcap_{i \in I} \lesssim^{i}$. Furthermore, it is obvious that $\lesssim=\bigcap_{\Sigma_{0} \in|\operatorname{Sign}|} \bigcap_{\phi \Sigma_{\Sigma_{0}} \psi} \lesssim\left\langle\Sigma_{0}, \phi, \psi\right\rangle$.

Thus, by Proposition $1,\langle\mathrm{SEN}, \lesssim\rangle$ is order isomorphic to an order subdirect product of the $\rho^{\left.\sim \Sigma_{0}, \phi, \psi\right\rangle}$-pofunctors $\langle\operatorname{SEN}, \lesssim\rangle / \lesssim\left\langle\Sigma_{0}, \phi, \psi\right\rangle, \Sigma_{0} \in|\operatorname{Sign}|, \phi, \psi \in \operatorname{SEN}\left(\Sigma_{0}\right)$, $\phi \mathbb{Z}_{\Sigma_{0}} \psi$.

To complete the proof, it now suffices to show that, for all $\Sigma_{0} \in|\operatorname{Sign}|$ and all $\phi, \psi \in \operatorname{SEN}\left(\Sigma_{0}\right)$, such that $\phi \mathbb{Z}_{\Sigma_{0}} \psi$, the $\rho^{\left.\sim \Sigma_{0}, \phi, \psi\right\rangle}$-pofunctor $\langle$ SEN, $\lesssim\rangle / \lesssim\left\langle\Sigma_{0}, \phi, \psi\right\rangle$ is order subdirectly irreducible. To this end, suppose that $\langle\mathrm{SEN}, \lesssim\rangle / \Sigma^{\left\langle\Sigma_{0}, \phi, \psi\right\rangle} \subseteq_{\mathrm{SD}} \prod_{i \in I}\left\langle\mathrm{SEN}^{\prime i}, \lesssim^{\prime i}\right\rangle$. Then, by Proposition 1 and the Order 
Correspondence Theorem (Theorem 19 of Voutsadakis, 2006), there exist $\lesssim^{i} \in \operatorname{QoSys}_{\rho}(\langle\mathrm{SEN}, \lesssim\rangle), i \in I$, such that $\lesssim\left\langle\Sigma_{0}, \phi, \psi\right\rangle=\bigcap_{i \in I} \lesssim^{i}$ and, for all $i \in I$, there exists a surjective order translation $\left\langle H^{i}, \gamma^{i}\right\rangle:\langle\mathrm{SEN}, \lesssim\rangle / \lesssim^{i} \rightarrow^{p}\left\langle\mathrm{SEN}^{\prime i}, \lesssim^{i}\right\rangle$, such that $\left\{H^{i}: i \in I\right\}$ are collectively mono and $H_{\Sigma}^{i}: \operatorname{SEN}^{\sim i}(\Sigma) \rightarrow \operatorname{SEN}^{\prime i}\left(H^{i}(\Sigma)\right)$ is an order isomorphism, for all $\Sigma \in \mid$ Sign|. But $\lesssim\left\langle\Sigma_{0}, \phi, \psi\right\rangle$ is completely meet-irreducible, whence we obtain that, there exists $i \in I$, such that $\lesssim_{\left.\Sigma_{0}, \phi, \psi\right\rangle}=\lesssim^{i}$, and, therefore, there also exists a surjective order translation $\left\langle H^{i}, \gamma^{i}\right\rangle:\langle\mathrm{SEN}, \lesssim\rangle / \Sigma^{\left\langle\Sigma_{0}, \phi, \psi\right\rangle} \rightarrow^{p}$ $\left\langle\operatorname{SEN}^{\prime i}, \lesssim^{\prime i}\right\rangle$, such that $\gamma_{\Sigma}^{i}: \operatorname{SEN}(\Sigma) / \sim_{\Sigma}^{\left\langle\Sigma_{0}, \phi, \psi\right\rangle} \rightarrow \operatorname{SEN}^{\prime i}\left(H^{i}(\Sigma)\right)$ is an order isomorphism, for all $\Sigma \in|\operatorname{Sign}|$. Thus, for all $\Sigma_{0} \in|\operatorname{Sign}|$ and all $\phi, \psi \in \operatorname{SEN}\left(\Sigma_{0}\right)$, such that $\phi \mathbb{Z}_{\Sigma_{0}} \psi$, the $\rho^{\sim\left\{\Sigma_{0}, \phi, \psi\right\rangle}$-pofunctor $\langle\mathrm{SEN}, \lesssim\rangle / \lesssim\left\langle\Sigma_{0}, \phi, \psi\right\rangle$ is order subdirectly irreducible.

\section{CLOSURE PROPERTIES OF QOSYs $\mathrm{s}_{\rho}^{\mathrm{K}}(\langle\mathrm{SEN}, \lesssim\rangle)$}

Suppose, now, that $\mathrm{K}$ is a class of pofunctors with compatible categories of natural transformations on their sentence functors and compatible polarities for them. For each $\rho$-pofunctor $\langle\mathrm{SEN}, \lesssim\rangle$, define

$$
\operatorname{QoSys}_{\rho}^{\mathrm{K}}(\langle\operatorname{SEN}, \lesssim\rangle)=\left\{\lesssim^{\prime} \in \operatorname{QoSys}_{\rho}(\langle\mathrm{SEN}, \lesssim\rangle):\langle\mathrm{SEN}, \lesssim\rangle / \lesssim^{\prime} \in \mathrm{K}\right\} .
$$

The qosystems in $\operatorname{QoSys}_{\rho}^{\mathrm{K}}(\langle\mathrm{SEN}, \lesssim\rangle)$ are referred to as the $\mathrm{K}$ - $\rho$-qosystems of $\langle\mathrm{SEN}, \lesssim\rangle$.

The following proposition is an analog in the context of partially ordered algebraic systems of Proposition 3.9 of Pigozzi (Preprint) for partially ordered algebras.

Proposition 3. Let $\mathrm{K}$ be a class of pofunctors, with compatible categories of natural transformations on their sentence functors and compatible polarities for them, and 〈SEN, \〉 a pofunctor, that is not necessarily in $\mathrm{K}$, but with a compatible category $N$ of natural transformations on SEN and a compatible polarity $\rho$ for $N$ with those of the pofunctors in $\mathrm{K}$.

1. If $\mathbf{P}_{\mathrm{SD}}(\mathrm{K}) \subseteq \mathrm{K}$, then $\operatorname{QoSys}_{\rho}^{\mathrm{K}}(\langle\mathrm{SEN}, \lesssim\rangle)$ is closed under arbitrary intersections and, hence, it contains a smallest $\mathrm{K}$ - $\rho$-qosystem.

2. If $\mathbf{H}(\mathrm{K}) \subseteq \mathrm{K}, \lesssim^{\prime} \in \operatorname{QoSys}_{\rho}^{\mathrm{K}}(\langle\mathrm{SEN}, \lesssim\rangle), \lesssim^{\prime \prime} \in \operatorname{QoSys}_{\rho}(\langle\mathrm{SEN}, \lesssim\rangle)$, with $\lesssim^{\prime} \leq \lesssim^{\prime \prime}$, then $\lesssim " \in \operatorname{QoSys}_{\rho}^{\mathrm{K}}(\langle\mathrm{SEN}, \lesssim\rangle)$.

3. If both $\mathbf{P}_{\mathrm{SD}}(\mathrm{K}) \subseteq \mathrm{K}$ and $\mathbf{H}(\mathrm{K}) \subseteq \mathrm{K}$ hold, then $\operatorname{QoSys}_{\rho}^{\mathrm{K}}(\langle\mathrm{SEN}, \lesssim\rangle)$ is a principal filter of $\mathbf{Q O S y s}_{\rho}(\langle\mathrm{SEN}, \lesssim\rangle)$.

Proof. 1. Suppose that $\left\{\lesssim^{i}: i \in I\right\} \subseteq \operatorname{QoSys}_{\rho}^{\mathrm{K}}(\langle\mathrm{SEN}, \lesssim\rangle)$. By Proposition 1 and the Order Correspondence Theorem (Theorem 19 of Voutsadakis, 2006), $\langle\mathrm{SEN}, \lesssim\rangle / \bigcap_{i \in I} \lesssim^{i}$ is order isomorphic to an order subdirect product of the collection $\langle\mathrm{SEN}, \lesssim\rangle / \lesssim^{i}, i \in I$. But, by hypothesis, for all $i \in I$, $\langle\mathrm{SEN}, \lesssim\rangle / \lesssim^{i} \in \mathrm{K}$, and $\mathbf{P}_{\mathrm{SD}}(\widetilde{\mathrm{K}}) \subseteq \mathrm{K}$, whence $\langle\mathrm{SEN}, \lesssim\rangle / \bigcap_{i \in I} \lesssim^{i} \in \mathrm{K}$. This shows that $\bigcap_{i \in I} \lesssim^{i} \in \operatorname{QoSys}_{\rho}^{\mathrm{K}}(\langle\mathrm{SEN}, \lesssim\rangle)$.

2. Now suppose that $\mathbf{H}(\mathrm{K}) \subseteq \mathrm{K}$ and

$$
\lesssim^{\prime} \in \operatorname{QoSys}_{\rho}^{\mathrm{K}}(\langle\mathrm{SEN}, \lesssim\rangle), \quad \lesssim^{\prime \prime} \in \operatorname{QoSys}_{\rho}(\langle\operatorname{SEN}, \lesssim\rangle),
$$


with $\lesssim \leq \lesssim^{\prime}$. The Order Homomorphism Theorem (Theorem 15 of Voutsadakis, 2006), gives that $\langle$ SEN, $\lesssim\rangle / \lesssim^{\prime \prime}$ is a homomorphic image of $\langle$ SEN, $\lesssim\rangle / \lesssim^{\prime}$. Moreover, by the hypothesis, we have $\langle\mathrm{SEN}, \lesssim\rangle / \lesssim^{\prime} \in \mathrm{K}$ and $\mathbf{H}(\mathrm{K}) \subseteq \mathrm{K}$, whence it follows that $\langle\mathrm{SEN}, \lesssim\rangle / \lesssim^{\prime \prime} \in \mathrm{K}$ and, therefore $\lesssim^{\prime \prime} \in \mathrm{QoSys}_{\rho}^{\mathrm{K}}(\langle\mathrm{SEN}, \lesssim\rangle)$.

3. This part follows from Parts 1 and 2 .

In forthcoming work, the results established in the present article will be exploited to provide analogs of Birkhoff's HSP Theorem, characterizing varieties of universal algebras, and Mal'cev's $\mathbf{S P P}_{\mathrm{U}}$ Theorem, characterizing quasi-varieties of universal algebras, in the context of pofunctors. Analogs of these two universal algebraic closure operators in the context of pofunctors have already been studied in Voutsadakis (Preprint b), where they have both been shown to be closure operators on classes of pofunctors.

\section{ACKNOWLEDGMENTS}

The author wishes to thank Katarzyna Pałasińska and Don Pigozzi, whose joint work presented in Pigozzi (Preprint) inspired and guided the work on partially ordered algebraic systems. To Don I owe a lot in terms of inspiration and support throughout the last decade.

\section{REFERENCES}

Barr, M., Wells, C. (1999). Category Theory for Computing Science. 3rd ed. Montréal: Les Publications CRM.

Blok, W. J., Pigozzi, D. (1986). Protoalgebraic logics. Studia Logica 45:337-369.

Blok, W. J., Pigozzi, D. (1989). Algebraizable logics. Memoirs of the American Mathematical Society 77:396.

Bloom, S. L. (1976). Varieties of ordered algebras. J. Computing and Systems Sciences 13:200-212.

Borceux, F. (1994). Handbook of Categorical Algebra. Encyclopedia of Mathematics and its Applications, Vol. 50. Cambridge, U.K.: Cambridge University Press.

Burris, S., Sankappanavar, H. P. (1981). A Course in Universal Algebra. New York: Springer-Verlag.

Czelakowski, J. (2001). Protoalgebraic Logics. Studia Logica Library 10. Dordrecht: Kluwer.

Dellunde, P. (1999). Equality-free logic: The method of diagrams and preservation theorems. Logic Journal of the IGPL 7:717-732.

Dellunde, P., Jansana, R. (1996). Some characterization theorems for infinitary universal horn logic without equality. J. Symbolic Logic 61:1242-1260.

Dunn, J. M. (1991). Gaggle theory: An abstraction of galois connections and residuations with applications to various logical operations. Logics in AI, Proceedings European Workshop JELIA 1990. Lecture Notes in Computer Science. No. 478. Springer-Verlag, pp. 31-51.

Dunn, J. M. (1993). Partial gaggles applied to logic with restricted structural rules. In: Schröder-Heiser, P., Došen, K., eds. Substructural Logics. Oxford: University Press, pp. 63-108.

Elgueta, R. (1997). Characterizing classes defined without equality. Studia Logica 58:357-394. Elgueta, R. (1998). Subdirect representation theory for classes without equality. Algebra Universalis 40:201-246. 
Font, J. M., Jansana, R. (1996). A General Algebraic Semantics for Sentential Logics. Lecture Notes in Logic, Vol. 7. Berlin-Heidelberg: Springer-Verlag.

Font, J. M., Jansana, R., Pigozzi, D. (2003). A survey of abstract algebraic logic. Studia Logica 74(1/2):13-97.

Fuchs, L. (1963). Partially Ordered Algebraic Structures. New York: Pergamon Press.

Mac Lane, S. (1971). Categories for the Working Mathematician. Springer-Verlag.

Mal'cev, A. I. (1966). Several remarks on quasi-varieties of algebraic systems. Algebra and Logic 5(3):3-9.

Mal'cev, A. I. (1973). Algebraic Systems. New York-Heidelberg: Springer-Verlag.

McKenzie, R. N., McNulty, G. F., Taylor, W. F. (1987). Algebras, Lattices, Varieties, Volume I. Monterey, California: Wadsworth \& Brooks/Cole.

Pigozzi, D. Partially Ordered Varieties and QuasiVarieties. Preprint available at http://www.math.iastate.edu/dpigozzi/

Voutsadakis, G. (2005a). Categorical abstract algebraic logic: models of $\pi$-institutions. Notre Dame Journal of Formal Logic 46(4):439-460.

Voutsadakis, G. (2005b). Categorical abstract algebraic logic: $(\mathscr{T}, N)$-algebraic systems. Applied Categorical Structures 13(3):265-280.

Voutsadakis, G. (2006). Categorical abstract algebraic logic: partially ordered algebraic systems. Applied Categorical Structures 14(1):81-98.

Voutsadakis, G. Categorical abstract algebraic logic: Tarski congruence systems, logical morphisms and logical quotients. Submitted to the Annals of Pure and Applied Logic. Preprint available at http://www.voutsadakis.com/RESEARCH/papers.html

Voutsadakis, G. Categorical abstract algebraic logic: closure operators on classes of pofunctors. Submitted to Algebra Universalis. Preprint available at http://www. voutsadakis.com/RESEARCH/papers.html 